\title{
Hashimoto encephalopathy: A study of the clinical profile, radiological and electrophysiological correlation in a Tertiary Care Center in South India
}

Pattanagere Manjunatha Suryanarayana Sharma, Mahendra Javali, Rohan Mahale, Byadarahalli Kempegowda Madhusudhan, Anas Abdul Majeed, Rangasetty Srinivasa

Department of Neurology, M.S. Ramaiah Medical College, Bengaluru, Karnataka, India

\section{ABSTRACT}

Background: Hashimoto encephalopathy (HE) is a poorly understood and often misdiagnosed entity with variable clinical spectrum. There are many uncertainties that still remain about this condition and the pathological significance of thyroid peroxidase (TPO) antibody. Objective: To characterize the clinical, laboratory and radiologic findings in patients with HE. Design: Retrospective analysis of clinical features and diagnostic test data. Main Outcome Measures: Clinical features, laboratory, radiologic, electroencephalography (EEG) findings associated with HE and therapeutic outcome. Results: Thirteen consecutive patients were identified as having HE. The median age at onset was 48.5 years (range, $19-62$ years). There was a female preponderance $(76.9 \%)$. Clinical manifestations were cognitive impairment and behavioral changes in $10(76.9 \%)$, sleep disturbance in $9(69.2 \%)$, seizures in $6(46.1 \%)$, headache in $4(30.8 \%)$, psychosis or paranoia in $5(38.5 \%)$, transient symptoms in $6(46.1 \%)$, myoclonus in $4(30.8 \%)$, ataxia or gait disorder in 4 (30.8\%). The most frequent laboratory abnormalities were increased TPO $(n=13)$ in all cases, increased thyroid stimulating hormone levels $(n=6)$, and increased erythrocyte sedimentation rate $(n=5)$. The cerebrospinal fluid protein level was elevated in 8 of 9 patients $(88.8 \%)$. Magnetic resonance imaging abnormalities were present in 2 patients (15.4\%). EEG changes were seen in 7 patients $(53.8 \%)$. All but two patients showed significant therapeutic benefit with steroids. Conclusions: HE has a wide range of clinical, laboratory, and radiologic findings. All patients with an unexplained encephalopathy should be screened for this condition as treatment response is excellent. To the best of our knowledge, this is the largest single center clinical series of HE from the Indian subcontinent.

Key words: Autoimmune encephalopathy, cognitive decline, Hashimoto encephalopathy, steroids, thyroid peroxidase, thyroid stimulating hormone

\section{Introduction}

Clinical profiles of acute or subacute encephalopathies are variable with diverse etiologies. Most often, the clinical features, laboratory investigations, electroencephalography (EEG), cerebrospinal fluid (CSF) and neuroimaging studies reveal an underlying cause for the encephalopathy. Once the infectious and metabolic causes for encephalopathy

\begin{tabular}{|l|l|}
\hline \multicolumn{2}{|c|}{ Access this article online } \\
\hline Quick Response Code: & Website: \\
\hline & www.ruralneuropractice.com \\
\cline { 2 - 3 } & \\
\hline & DOI: \\
\hline ⿴囗口 & $10.4103 / 0976-3147.158753$ \\
\hline
\end{tabular}

are ruled out, an inflammatory or autoimmune cause should be considered. Autoimmune encephalopathy has various forms including that against known pathogenic antigens (e.g.: Voltage-gated potassium channel (VGKC)-complex, N-methy D-aspartate receptor [NMDAR] etc.) and also idiopathic autoimmune encephalopathy responding to steroids termed as Hashimoto encephalopathy (HE) or steroid responsive encephalopathy associated with autoimmune thyroiditis. HE is a rare, autoimmune disease characterized by encephalopathy and elevated antithyroid antibodies in the absence of a central nervous system (CNS) infection, tumor or stroke. ${ }^{[1]} \mathrm{HE}$ was postulated to be a distinct disease entity by Brain et al. in 1966. ${ }^{[2]}$ It is a disease of the CNS having a good prognosis if diagnosed and treated early. ${ }^{[3]}$ The clinical symptoms are nonspecific, the onset being acute, subacute or

\section{Address for correspondence:}

Dr. R. Srinivasa, Department of Neurology, No. 107, First Floor, M. S. Ramaiah Memorial Hospital, M. S. R. I. T. Post, New B. E. L. Road, Bengaluru - 560 054, Karnataka, India. E-mail: pmssharma17@gmail.com 
chronic with a variable disease course-self-limiting, progressive or relapsing-remitting. ${ }^{[4]}$ The symptoms can remit spontaneously or after corticosteroids. There are frequently reported misdiagnoses of this disease in the literature. ${ }^{[5]}$ Clinical presentations of HE ranges from amnestic syndrome, ${ }^{[6]}$ seizures including status epilepticus, ${ }^{[7]}$ ataxia $^{[8]}$ myoclonus $^{[9]}$ and psychiatric manifestations including depression, ${ }^{[10]}$ mania, ${ }^{[11]}$ psychosis and hallucinations. ${ }^{[12,13]}$ This entity has attracted growing attention because it is included in the group of treatable dementias. ${ }^{[14]}$ There are case reports, small series and literature reviews that have made an attempt to characterize further this entity. ${ }^{[15-17]}$ Nevertheless, there are many uncertainties that still remain about this condition including its clinical spectrum, laboratory and radiological findings and the significance of quantitative levels of thyroid peroxidase (TPO) antibody. The present study is an attempt to analyse data on a series of patients in whom the diagnosis of $\mathrm{HE}$ was made during a 31/2 years period.

\section{Subjects and Methods}

This is a retrospective, hospital-based study. The study was approved by the institutional scientific committee and ethics review board. The hospital registry was screened to identify records with a diagnosis of encephalopathy from January 2010 to June 2013. 675 patient records were identified.

The following criteria was used for the diagnosis of HE: (a) Acute or subacute onset of altered mental status (AMS), (b) elevated antithyroid antibodies (c) rapid response in mental status with corticosteroids and (d) absence of structural, infectious or other metabolic factors which could explain the AMS and its response to steroids. ${ }^{[18]}$ The exclusion criteria for the study were (a) Illness attributable to infective/ metabolic/toxic/vascular etiology, (b) Illness attributable to structural lesion/traumatic brain injury, (c) postoperative encephalopathy and (d) age of patients $<18$ years. Out of the 675 patients screened, 29 fulfilled the diagnosis of encephalopathy with high TPO antibody levels (>60 IU/mL). 16 patients were excluded as per the exclusion criteria. 13 patients were found to meet the defined criteria for HE. The case records of the included patients were reviewed in detail. Data on age, gender, clinical presentation, past medical history, medications, antithyroid antibodies levels, brain magnetic resonance imaging (MRI), EEG, CSF analysis, treatment, and outcome were collected. Statistical analysis was done using mean, median, standard deviation and percentage.

\section{Results}

\section{Clinical findings}

Thirteen patients fulfilled criteria for the diagnosis of HE. The clinical profile of 13 patients is given in Table 1. All but two patients required hospitalization because of the severity of their deficits. The median age at onset was 48.5 years (range, 19-62 years). There was a preponderance of female patients $(76.9 \%)$. The most common associated manifestations were cognitive impairment and behavioral changes in 10 (76.9\%), mean MMSE 22 (range: 16-28) patients had predominantly frontal and temporal lobe involvement on neuropsychological evaluation, seizures in $6(46.1 \%)$, sleep disturbance in $9(69.21 \%)$ in the form of hypersomnia in 6 and reduced sleep in 3 , headache in $4(30.8 \%)$ patients. The most frequent alternate diagnoses at admission were acute encephalitis in $3(23.1 \%)$, dementia in $2(15.4 \%)$, psychosis in $2(15.4 \%)$, anxiety disorder in $2(15.4 \%)$, secondary GTCS in $2(15.4 \%)$ and status epilepticus in $01(7.7 \%)$ patient. Five patients had a history of hypothyroidism that antedated the onset of neurologic symptoms, and 1 had euthyroid goiter. Four additional patients developed hypothyroidism after the resolution of encephalopathy. Other associated conditions in 6 patients included diabetes in $3(23.1 \%)$, Vitamin B12 deficiency in $1(7.7 \%)$, systemic lupus erythematosus in $1(7.7 \%)$ and seizures in $1(7.7 \%)$. Five patients $(38.5 \%)$ had a family history of thyroid illness.

\section{Laboratory tests}

By definition, all patients had thyroid antibodies. The TPO antibody titer was elevated in all 13 patients tested (median, $909 \mathrm{IU} / \mathrm{mL}$; range, $82-1300 \mathrm{IU} / \mathrm{mL}$; reference range, $0-34 \mathrm{IU} / \mathrm{mL}$ ). Five patients had mild thyroid failure (serum thyroid stimulating hormone, median 7.22 $\mu \mathrm{IU} / \mathrm{L}$, range 0.858-22.1 $\mu \mathrm{IU} / \mathrm{L}$ ). Erythrocyte sedimentation rate was mildly elevated (median, $14.7 \mathrm{~mm} / \mathrm{h}$; range, $2-35 \mathrm{~mm} / \mathrm{h})$ in $5(38.7 \%)$ patients. The C-reactive protein level was elevated in 3 (23.1\%) patients. Liver aminotransferase levels (aspartate aminotransferase/alanine aminotranferease) were mildly elevated in $3(23.1 \%)$ patients. Antinuclear antibody profile was negative in 4 cases. VGKC/anti-NMDAR antibodies were negative in 4 cases. Pertinent laboratory findings are given in Table 2.

\section{Cerebrospinal fluid analysis}

The CSF was analyzed in 9 out of the 13 patients. 4 patients refused to give consent for a lumbar puncture. No infectious etiology was identified. The protein level was elevated in 8 of 9 patients (88.8\%) (Median: $51.45 \mathrm{mg} / \mathrm{dL}$, range, $45.3-66.5 \mathrm{mg} / \mathrm{dL}$; reference range: $0-45 \mathrm{mg} / \mathrm{dL}$ ). Only two patients had mild lymphocytic pleocytosis with 6 and 8 cells each. 
Table 1: Clinical findings in thirteen patients with Hashimoto encephalopathy

\begin{tabular}{lc}
\hline Abnormal clinical finding & $\begin{array}{c}\text { Number }(\%) \text { of } \\
\text { patients }(\boldsymbol{n}=13)\end{array}$ \\
\hline Cognitive impairment and behavioral changes & $10(76.9)$ \\
Sleep disturbance & $09(69.2)$ \\
Hyper somnolence & $03(23.1)$ \\
Insomnia & $06(46.1)$ \\
Fluctuating symptoms & $06(46.1)$ \\
Psychosis or paranoia & $05(38.5)$ \\
Myoclonus & $04(30.8)$ \\
Ataxia or gait disorder & $04(30.8)$ \\
Headache & $04(30.1)$ \\
Seizures & $06(46.1)$ \\
Generalized & $04(30.8)$ \\
Partial & $01(7.7)$ \\
Both & $01(7.7)$ \\
Tremors & $01(7.7)$ \\
\hline
\end{tabular}

Table 2: Serologic and other laboratory abnormalities in 13 patients with Hashimoto's encephalopathy

\begin{tabular}{lc}
\hline Serologic or laboratory test & $\begin{array}{c}\text { Number of } \\
\text { patients* }\end{array}$ \\
\hline Thyroperoxidase antibody positive & $13 / 13$ \\
Thyroglobulin antibody positive & $01 / 02$ \\
ANA positive & $04 / 13$ \\
Rheumatoid factor positive & $02 / 13$ \\
ESR elevated & $05 / 13$ \\
CRP level elevated & $03 / 13$ \\
Liver aminotransferase levels (AST/ALT) elevated & $03 / 13$ \\
VGKC/NMDAR antibody negative & $04 / 04$ \\
CSF protein elevated & $08 / 09$ \\
CSF cell elevated (>5 cells/cu.mm) & $02 / 09$ \\
\hline
\end{tabular}

${ }^{*}$ Depicts number of patients with abnormal tests against total number of patients in whom it was performed. CSF: Cerebrospinal fluid,

ALT: Alanine aminotranferease, AST: Aspartate aminotransferase, VGKC/ NMDAR: Voltage gated potassium channel-complex/N-methy D-aspartate receptor, CRP: C-reactive protein, ESR: Erythrocyte sedimentation rate, ANA: Antinuclear antibody

\section{Electroencephalography abnormalities}

All patients underwent EEG studies, of which 7 results were abnormal. Generalized slowing was seen in 7 patients, one such slowing is shown in Figure 1. Other findings included focal slowing, triphasic waves, epileptiform abnormalities, and photic stimulation induced discharges. Six patients had a normal routine EEG results.

\section{Neuroimaging}

Cranial MRI was performed in 13 patients. Ten patients $(76.8 \%)$ had normal imaging results or nonspecific white matter abnormalities consistent with age. MRI scan of patient no. 12 is shown in Figure 2. Two patients had a diffuse increased signal on T2-weighted and fluid-attenuated inversion recovery images in the cerebral white matter, and 1 MRI revealed mild ventriculomegaly.

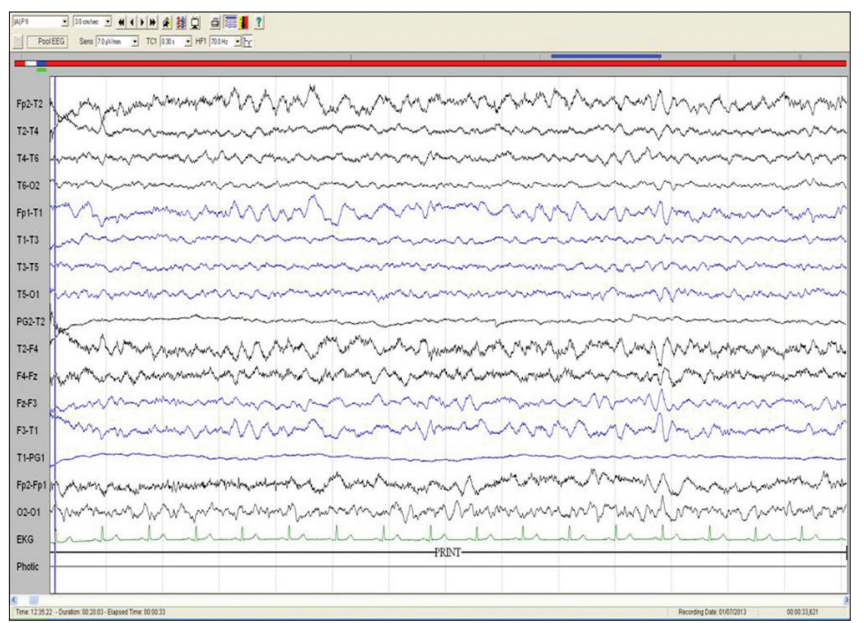

Figure 1: Electroencephalography of patient no. 7: Bipolar longitudinal montage (sensitivity $7.5 \mu \mathrm{V} / \mathrm{mm}$, low frequency filter $1 \mathrm{~Hz}$, high frequency filter $70 \mathrm{~Hz}$, notch, $50 \mathrm{HZ}$, speed $30 \mathrm{~mm} / \mathrm{s}$ ) showing diffuse slowing of background rhythm (frequency: $3-5 \mathrm{~Hz}$ )

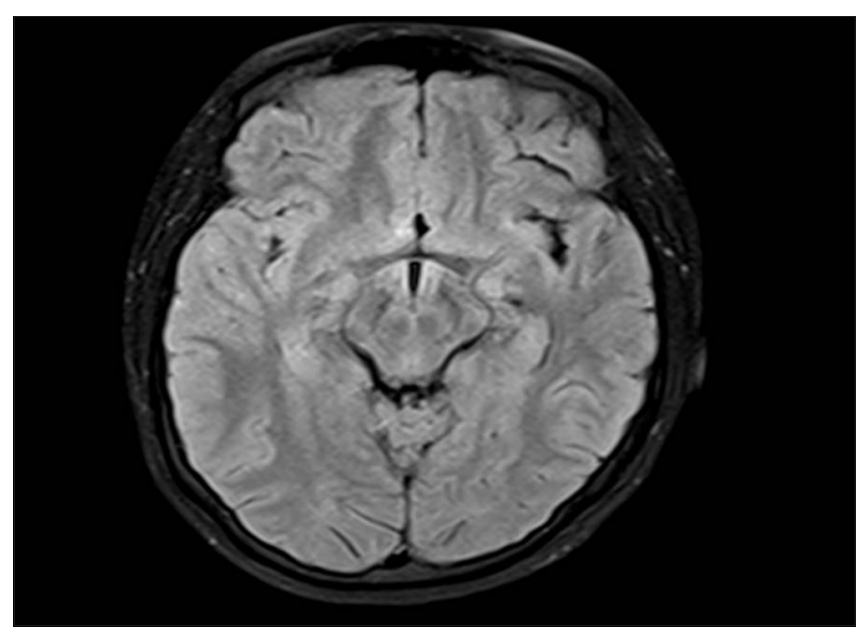

Figure 2: Magnetic resonance imaging fluid-attenuated inversion recovery axial image of patient no. 12 showing normal brain parenchyma

\section{Therapy and clinical outcome during follow-up}

Intravenous methyl prednisolone, $1 \mathrm{~g} / \mathrm{d}$ for 5 days, was used in 10 patients followed by a course of oral steroids in 9 patients. Oral prednisone was given at $1 \mathrm{mg} / \mathrm{kg} / \mathrm{d}$ with slow taper over 10-30 days in 2 patients. After treatment 8 patients had returned to their normal neurologic baseline status, 2 had mild residual symptoms, and 3 patients were lost for follow-up. Residual symptoms included gait impairment and mild forgetfulness in one patient each. Nine patients were able to discontinue the use of steroids after initial treatment without any subsequent relapses. Three patients were lost to follow-up. Thyroid hormone therapy was initiated in patients with laboratory evidence of hypothyroidism, but the neurological improvement did not correlate with the hormone replacement. Antiepileptics were given in 4 cases. 


\section{Discussion}

Hashimoto encephalopathy is a rare disease with an estimated prevalence of 2.1/100,000. ${ }^{[19]}$ The diagnosis of HE should be considered in patients with no prior neurological problems presenting with a well-defined encephalopathy. The characteristics of patients in this case series may help in improving the recognition of this condition and instituting timely therapy. The clinical features are varied but are consistent with those reported previously. ${ }^{[1]}$ Cognitive impairment along with behavioral changes was the defining characteristic of $\mathrm{HE}$ in the present case series (76.4\%). Other clinical features that characterize HE were transient symptoms in the form of the sensory phenomenon, aches and cramps. Patients also had myoclonus, sleep abnormalities, seizures, headache, psychosis and gait difficulties. Due to the nonspecific nature of the neurological and laboratory findings of $\mathrm{HE}$, it is not surprising that alternative initial diagnoses were considered before arriving at a definite diagnosis. Thyroid antibody levels in the present series were variable. Most of the patients had cognitive impairment and psychiatric features, but antibody levels did not correspond to the severity of the clinical deficits suggesting that HE should be considered in patients with encephalopathy irrespective of thyroid antibody levels. A review of 85 patients had shown that $38 \%$ had normal thyroid function at presentation, $35 \%$ had subclinical hypothyroidism, $20 \%$ had overt hypothyroidism and $7 \%$ had hyperthyroidism. ${ }^{[1]}$ Furthermore, HE should be considered in patients with encephalopathy regardless of thyroid function status as many are euthyroid. The presence of elevated CSF protein levels in most of our patients ( 8 out of 13 ) suggesting an underlying inflammatory process is consistent with prior reports. ${ }^{[20]}$ However, it is important to remember that normal CSF protein levels or absence of lymphocytic pleocytosis do not exclude the diagnosis of HE. Most patients (76.8\%) with the diagnosis of HE had normal neuroimaging results. Only two patients had diffuse white matter changes. The imaging characteristics in HE are different from the striking mesial temporal lobe abnormalities encountered in many patients with subacute inflammatory autoimmune or paraneoplastic limbic encephalitis. ${ }^{[21]}$

The exact role of thyroid autoimmunity in the pathogenesis of HE is complicated by the fact that serum TPO levels are elevated in approximately $10 \%$ of healthy adults, and the prevalence of individuals with elevated TPO levels increases with increasing age. ${ }^{[22]}$ Thyroid autoantibodies are also commonly found in patients with other autoimmune neurologic disorders, including paraneoplastic and nonparaneoplastic limbic encephalitis. ${ }^{[23]}$ It, therefore, seems unlikely that thyroid antibodies are the direct cause of the encephalopathy. Apart from hypothyroidism, there are several other etiologies that have been proposed, including humoral factors, antigen-antibody complexes, overt vasculitis, intrathecal thyroid antibodies, and global cerebral hypoperfusion. ${ }^{[24-26]}$

More than one etiologic mechanism is responsible for autoimmune or inflammatory encephalopathy that responds to corticosteroids. Systematic studies of patients with encephalopathy, which could include a battery of serum and CSF autoimmune markers, may offer insights into the pathogenetic mechanisms.

Marked clinical improvement was seen in all 13 patients with high-dose corticosteroid therapy in the present study. Similar improvement with corticosteroid therapy was seen in the study by Castillo et al. in 2013. ${ }^{[5]}$ Comparison of the present study with other 2 published case series on HE is given in Table 3. Patients resistant to steroid therapy may benefit by other immunomodulation therapies such as intravenous immunoglobulin/plasma exchange as reported by Jacob et al. in 2008.

We acknowledge several limitations of this study. Because of the retrospective nature of this case series, not all patients underwent the same tests and evaluations. VGKC/NMDAR antibodies were done only in last 4 cases as the testing facility was not available before that period. The data presented in this series should not be viewed as showing the entire spectrum of neurologic and laboratory findings associated with HE. Antithyroid antibody levels in CSF and during follow-up were not done in all patients. Being a hospital-based study; it is not a true representation of the prevalence of the disease in the community.

However, we are hopeful that this study findings will improve the recognition rate of patients who may have an autoimmune or inflammatory mechanism underlying their encephalopathy, as well as stimulate further research into HE.

\section{Conclusions}

Hashimoto encephalopathy has a wide range of clinical, laboratory, and radiologic findings. All patients with an unexplained acute/subacute/chronic encephalopathy should be screened HE as the therapeutic response is excellent. Increased awareness of this clinical entity among physicians, endocrinologists and neurophysicians 
Table 3: Comparison of clinical and laboratory data with other published case series

\begin{tabular}{|c|c|c|c|}
\hline Findings & Present series $(n=13)$ & Castillo et al. $2006(n=20)$ & Olmez et al. $2013(n=13)$ \\
\hline Median age at onset (years) & $48.5(19-62)$ & $56(27-84)$ & $49(2-66)$ \\
\hline Gender (female) (\%) & $10(77)$ & $14(70)$ & $12(92)$ \\
\hline \multicolumn{4}{|l|}{ Clinical findings (\%) } \\
\hline Cognitive impairment/behavioral changes & $10(76.9)$ & $20(100)$ & $8(61)$ \\
\hline Transient symptoms & $6(46.1)$ & $19(95)$ & $5(38)$ \\
\hline Tremor & $1(7.7)$ & $16(80)$ & $3(23)$ \\
\hline Myoclonus & $4(30.8)$ & $13(65)$ & $5(38)$ \\
\hline Ataxia or gait disorder & $4(30.8)$ & $13(65)$ & $2(15)$ \\
\hline Seizures & $6(46.1)$ & $12(60)$ & $7(53)$ \\
\hline Generalized & $4(30.8)$ & $10(50)$ & $5(38)$ \\
\hline Partial & $1(7.7)$ & $7(35)$ & $1(8)$ \\
\hline Both & $1(7.7)$ & $5(25)$ & $1(8)$ \\
\hline Sleep disturbance & $9(46.1)$ & $11(55)$ & $6(46)$ \\
\hline Hyper somnolence & $3(23.1)$ & $8(40)$ & $2(15)$ \\
\hline Insomnia & $6(46.1)$ & $3(15)$ & $4(31)$ \\
\hline Headache & $4(30.1)$ & $10(50)$ & $3(23)$ \\
\hline Lateralized motor or sensory deficits & $0(0)$ & $5(25)$ & $2(15)$ \\
\hline Psychosis or paranoia & $5(38.5)$ & $5(25)$ & $2(15)$ \\
\hline \multicolumn{4}{|l|}{ Serologic abnormalities } \\
\hline Thyroperoxidase antibody positive & $13 / 13$ & $20 / 20$ & $13 / 13$ \\
\hline Thyroglobulin antibody positive & 01/02 & $06 / 10$ & $11 / 11$ \\
\hline ANA positive & $04 / 13$ & $06 / 20$ & NA \\
\hline RA factor positive & $02 / 13$ & $01 / 17$ & NA \\
\hline ESR elevation & $05 / 13$ & $05 / 19$ & NA \\
\hline CRP level elevated & 03/03 & $3 / 9$ & NA \\
\hline AST/ALT elevated & 03/03 & $11 / 20$ & NA \\
\hline TSH & $05 / 13$ & $11 / 20$ & $5 / 10$ \\
\hline \multicolumn{4}{|l|}{ CSF abnormalities } \\
\hline Protein level elevated & $08 / 13$ & $17 / 20$ & $4 / 10$ \\
\hline Leukocyte count elevated $(>5)$ & $02 / 13$ & $05 / 20$ & $3 / 10$ \\
\hline
\end{tabular}

TSH: Thyroid stimulating hormone, CSF: Cerebrospinal fluid, ALT: Alanine aminotranferease, AST: Aspartate aminotransferase, CRP: C-reactive protein, ESR: Erythrocyte sedimentation rate, ANA: Antinuclear antibody, RA: Rheumatoid arthritis, NA: Not available

may help in early diagnosis and therapy with good clinical outcome. To the best of our knowledge, this is the largest single center clinical series of HE from the Indian subcontinent.

\section{Acknowledgment}

DR. Acharya P.T. formerly Professor, Department of neurology, M. S. Ramaiah Medical college for his valuable guidance.

Dr. A. S. Hegde, Director, M. S. Ramaiah Institute of Neurosciences for supervising the manuscript preparation and useful suggestions.

Dr. Sudhindra Aroor, Mrs. Hemavathi and Mrs. Gayathri for Secretarial assistance in preparation of the manuscript.

\section{References}

1. Chong JY, Rowland LP, Utiger RD. Hashimoto encephalopathy: Syndrome or myth? Arch Neurol 2003;60:164-71.
2. Brain L, Jellinek EH, Ball K. Hashimoto's disease and encephalopathy. Lancet 1966;2:512-4.

3. Boers PM, Colebatch JG. Hashimoto's encephalopathy responding to plasmapheresis. J Neurol Neurosurg Psychiatry 2001;70:132.

4. Mijajlovic M, Mirkovic M, Dackovic J, Zidverc-Trajkovic J, Sternic N. Clinical manifestations, diagnostic criteria and therapy of Hashimoto's encephalopathy: Report of two cases. J Neurol Sci 2010;288:194-6.

5. Castillo P, Woodruff B, Caselli R, Vernino S, Lucchinetti C, Swanson J, et al. Steroid-responsive encephalopathy associated with autoimmune thyroiditis. Arch Neurol 2006;63:197-202.

6. McCabe DJ, Burke T, Connolly S, Hutchinson M. Amnesic syndrome with bilateral mesial temporal lobe involvement in Hashimoto's encephalopathy. Neurology 2000;54:737-9.

7. McGinley J, McCabe DJ, Fraser A, Casey E, Ryan T, Murphy R. Hashimoto's encephalopathy; an unusual cause of status epilepticus. Ir Med J 2000;93:118.

8. Nakagawa H, Yoneda M, Fujii A, Kinomoto K, Kuriyama M. Hashimoto's encephalopathy presenting with progressive cerebellar ataxia. J Neurol Neurosurg Psychiatry 2007;78:196-7.

9. Arya R, Anand V, Chansoria M. Hashimoto encephalopathy presenting as progressive myoclonus epilepsy syndrome. Eur J Paediatr Neurol 2013;17:102-4.

10. Liu CY, Tseng MC, Lin PH. Encephalopathy associated with autoimmune thyroid disease (Hashimoto's thyroiditis) presenting as depression: A case report. Gen Hosp Psychiatry 2011;33:641.e7-9.

11. Lin ST, Chen CS, Yang P, Chen CC. Manic symptoms associated with Hashimoto's encephalopathy: Response to corticosteroid treatment. 
J Neuropsychiatry Clin Neurosci 2011;23:E20-1.

12. Wilcox RA, To T, Koukourou A, Frasca J. Hashimoto's encephalopathy masquerading as acute psychosis. J Clin Neurosci 2008;15:1301-4.

13. Mahmud FH, Lteif AN, Renaud DL, Reed AM, Brands CK. Steroid-responsive encephalopathy associated with Hashimoto's thyroiditis in an adolescent with chronic hallucinations and depression: Case report and review. Pediatrics 2003;112 (3 Pt 1):686-90.

14. Galluzzi S, Geroldi C, Zanetti O, Frisoni GB. Hashimoto's encephalopathy in the elderly: Relationship to cognitive impairment. J Geriatr Psychiatry Neurol 2002;15:175-9.

15. Sawka AM, Fatourechi V, Boeve BF, Mokri B. Rarity of encephalopathy associated with autoimmune thyroiditis: A case series from Mayo Clinic from 1950 to 1996. Thyroid 2002;12:393-8.

16. Kothbauer-Margreiter I, Sturzenegger M, Komor J, Baumgartner R, Hess CW. Encephalopathy associated with Hashimoto thyroiditis: Diagnosis and treatment. J Neurol 1996;243:585-93.

17. Cantón A, de Fàbregas $O$, Tintoré M, Mesa J, Codina A, Simó R. Encephalopathy associated to autoimmune thyroid disease: A more appropriate term for an underestimated condition? J Neurol Sci 2000;176:65-9.

18. Olmez I, Moses H, Sriram S, Kirshner H, Lagrange AH, Pawate S. Diagnostic and therapeutic aspects of Hashimoto's encephalopathy. J Neurol Sci 2013;331:67-71.

19. Ferracci F, Bertiato G, Moretto G. Hashimoto's encephalopathy: Epidemiologic data and pathogenetic considerations. J Neurol Sci 2004;217:165-8.

20. Seipelt M, Zerr I, Nau R, Mollenhauer B, Kropp S, Steinhoff BJ, et al.
Hashimoto's encephalitis as a differential diagnosis of Creutzfeldt-Jakob disease. J Neurol Neurosurg Psychiatry 1999;66:172-6.

21. Lawn ND, Westmoreland BF, Kiely MJ, Lennon VA, Vernino S. Clinical, magnetic resonance imaging, and electroencephalographic findings in paraneoplastic limbic encephalitis. Mayo Clin Proc 2003;78:1363-8.

22. Marcocci C, Chiovato L. Thyroid-directed antibodies. In: Braberman L, editor. The Thyroid. Vol. 8. Baltimore, MD: Williams and Wilkins; 2000. p. 414-31.

23. Thieben MJ, Lennon VA, Boeve BF, Aksamit AJ, Keegan M, Vernino S. Potentially reversible autoimmune limbic encephalitis with neuronal potassium channel antibody. Neurology 2004;62:1177-82.

24. Garrard P, Hodges JR, De Vries PJ, Hunt N, Crawford A, Hodges JR, et al. Hashimoto's encephalopathy presenting as "myxodematous madness". J Neurol Neurosurg Psychiatry 2000;68:102-3.

25. Ferracci F, Moretto G, Candeago RM, Cimini N, Conte F, Gentile M, et al. Antithyroid antibodies in the CSF: Their role in the pathogenesis of Hashimoto's encephalopathy. Neurology 2003;60:712-4.

26. Forchetti CM, Katsamakis G, Garron DC. Autoimmune thyroiditis and a rapidly progressive dementia: Global hypoperfusion on SPECT scanning suggests a possible mechanism. Neurology 1997;49:623-6.

How to cite this article: Pattanagere Manjunatha Suryanarayana Sharma, Mahendra Javali, Rohan Mahale, Byadarahalli Kempegowda Madhusudhan, Anas Abdul Majeed, Rangasetty Srinivasa. Hashimoto encephalopathy: A study of the clinical profile, radiological and electrophysiological correlation in a Tertiary Care Center in South India. J Neurosci Rural Pract 2015;6:309-14.

Source of Support: Nil. Conflict of Interest: None declared. 